\title{
Neutron Resonances: Widths Distribution versus the Porter-Thomas Law
}

\author{
V.V. SOKOLOV* \\ Budker Institute of Nuclear Physics of SB RAS, Novosibirsk, Russia
}

\begin{abstract}
The neutron resonance scattering off heavy nuclei is a paradigmatic example of the chaotic processes that are well described within the framework of the standard Random Matrix Theory (RMT). In zero approximation of non-overlapping resonances, the resonance width distribution is given by the standard Porter-Thomas law (PTD) $\frac{\mathrm{d} w}{\mathrm{~d} x}=\frac{\mathrm{e}^{-x / 2}}{\sqrt{2 \pi x}}$, where $x=\Gamma /\langle\Gamma\rangle$ is the resonance width measured in the units of its mean value. We analyze the influence of the resonance overlapping and show that the experimentally observed deviations from of the PTD arise due to the influence of a moderate number of neighboring resonances located inside a restricted energy interval within which the mean level spacing $D$ remains constant.
\end{abstract}

DOI: 10.12693/APhysPolA.132.1704

PACS/topics: $05.45 . \mathrm{Gg}, 24.60 . \mathrm{Lz}, 05.45 . \mathrm{Mt}$

\section{Introduction}

It has been announced recently [1] that a more careful analysis of old existing datasets as well as the new more precise experimental measurements demonstrate noticeable deviations of the neutron resonance widths distribution from the Porter-Thomas law. Actually, this is quite a misleading statement. Strictly speaking, the PTD is valid only if the resonance of the interest is not exposed to any influence of other ones. Obviously, as a rule this is not the case. In practice, neighboring resonances overlap because of their finite widths and this essentially influences on the width distribution of any of them.

We consider below a succession of a finite number $N=2,3, \ldots$ of resonance excitations that occupy some finite energy interval $a$ within which the mean level spacing $D$ remains constant. The joint probability density distribution of the resonance energies $E_{n}$ and corresponding widths $\Gamma_{n}$ of such a sequence has been rigorously derived in Ref. [2]. The internal dynamics was supposed to be chaotic and described by the Gaussian Orthogonal Ensemble (GOE) of $N \times N$ random matrices. As regards the amplitudes that connect the internal and channel states, they are assumed to be random Gaussian.

Actually, it is convenient to use the dimensionless energy variables defined as $\varepsilon_{n}=\frac{E_{n}}{D}$ and $\sigma=\frac{a}{D}$. The mentioned above joint probability density reads then as:

$$
\begin{aligned}
& P\left(\varepsilon_{1}, \ldots, \varepsilon_{N} ; x_{1}, \ldots, x_{N} ; \kappa\right)= \\
& C_{N}\left(\frac{1}{\sigma}\right)^{\frac{N(N+1)}{2}} \prod_{n}^{N} \exp \left(-\frac{N}{\sigma^{2}} \varepsilon_{n}^{2}\right) \\
& \quad \times \prod_{m<n}^{N} \frac{\left(\varepsilon_{n}-\varepsilon_{m}\right)^{2}+\frac{\kappa^{2}}{4}\left(x_{n}-x_{m}\right)^{2}}{\sqrt{\left(\varepsilon_{n}-\varepsilon_{m}\right)^{2}+\frac{\kappa^{2}}{4}\left(x_{n}+x_{m}\right)^{2}}}
\end{aligned}
$$

*e-mail: V.v.Sokolov@inp.nsk.su

$$
\times \exp \left(-\frac{N}{2 \sigma^{2}} \kappa^{2} \sum_{m<n} x_{m} x_{n}\right) \prod_{n}^{N} \frac{\mathrm{e}^{-\frac{1}{2} x_{n}}}{\sqrt{2 \pi x_{n}}}
$$

where the GOE normalization constant $C_{N}$ equals [3]

$$
C_{N}=2^{\frac{N(N-1)}{4}} \frac{N^{N(N+1) / 4}}{N !} \frac{1}{\prod_{n} \Gamma\left(\frac{n}{2}\right)} .
$$

The parameter $\sigma$ increases monotonously with the number $N$ of resonances. At last, the ratio $\kappa=\frac{\langle\Gamma\rangle}{D}$ controls the degree of the resonance overlapping.

\section{Non-overlapping resonances}

In the absence of the resonance overlapping, $\kappa=0$, the total joint distribution splits into a product of two independent distributions: of the energies $\varepsilon$

$$
\begin{aligned}
& P_{\varepsilon}\left(\varepsilon_{1}, \ldots, \varepsilon_{N} ; \kappa=0\right)= \\
& C_{N}\left(\frac{1}{\sigma}\right)^{\frac{N(N+1)}{2}} \prod_{m<n}^{N}\left|\varepsilon_{n}-\varepsilon_{m}\right| \exp \left(-\frac{N \sum_{n} \varepsilon_{n}^{2}}{\sigma^{2}}\right) .
\end{aligned}
$$

and widths

$$
P\left(x_{1}, \ldots, x_{N} ; \kappa=0\right)=\prod_{n}^{N} \frac{\exp \left(-\frac{1}{2} x_{n}\right)}{\sqrt{2 \pi x_{n}}} .
$$

The both these distributions are normalized to unity.

In the formal limit of infinitely large, $N \Rightarrow \mathbb{N} \rightarrow \infty$, number of (stable) energy levels, the standard RMT predicts the well known Wigner semicircle law:

$$
\rho(\varepsilon)=\frac{1}{D} \sqrt{1-\frac{\pi^{2}}{4 \mathbb{N}^{2}} \varepsilon^{2}},
$$

that is valid within the interval $-\frac{2 \mathbb{N}}{\pi} \leqslant \varepsilon \equiv \frac{E}{D} \leqslant \frac{2 \mathbb{N}}{\pi}$, where $D=\frac{\pi}{2 \mathbb{N}} \lambda$ is the mean level spacing at the center of a semicircle with a finite radius $\lambda$. The semicircle law predicts unrestrictedly increasing mean level spacing when the edges of the semicircle are approached. Such a behavior has nothing in common with the properties of the actual resonance spectra of heavy nuclei. However, the density of levels and, correspondingly, the mean level 
spacing $D$ remain constant within the interval

$$
-\frac{2 N}{\pi} \leqslant \varepsilon \leqslant \frac{2 N}{\pi}
$$

where a finite number $N \lll \mathbb{N}$ of energy levels are disposed. Below we concentrate our attention on the properties of such a finite chain of in average equidistant levels.

\section{Overlapping resonances}

Deviation of the width distribution of any arbitrarily chosen resonance state from the Porter-Thomas law is caused by mutual resonance overlapping $(\kappa>0)$. Well below the threshold of the super radiance transition [2] the overlap parameter is restricted to the interval $0<$ $\kappa<1$.

Two calculations have to be performed to get the width distribution of a chosen resonance:

1. Integration over all energies $\varepsilon_{n}$ provides the joint widths distribution:

$$
\begin{aligned}
& P\left(x_{1}, \ldots, x_{N} ; \kappa\right)=\Phi\left(x_{1}, \ldots, x_{N} ; \kappa\right) \\
& \quad \times \exp \left(-\frac{N}{2 \sigma^{2}} \kappa^{2} \sum_{m<n} x_{m} x_{n}\right) \prod_{n}^{N} \frac{\exp \left(-\frac{1}{2} x_{n}\right)}{\sqrt{2 \pi x_{n}}},
\end{aligned}
$$

2. Finally, integration over all widths excluding the one of our interest results in

$$
P(x ; \kappa)=\Psi(x ; \kappa) \frac{\exp \left(-\frac{1}{2} x\right)}{\sqrt{2 \pi x}} .
$$

Both the functions $\Phi$ and $\Psi$ satisfy the condition

$$
\Phi\left(x_{1}, \ldots, x_{N} ; 0\right)=\Psi(x ; 0)=1 .
$$

Just the factor $\Psi(x ; \kappa)$ describes deviation of the genuine neutron width distribution from the Porter-Thomas formula and is therefore the object of the prime interest.

Actually, the full analytical calculation of these functions is quite an intricate task. Mainly, some analytical results were obtained up to now in the limiting case of infinitely large number of resonances, [4-7]. Quite opposite, we show below that the principal influence on a chosen resonance comes from a restricted number of its near neighbors.

\section{Two simple examples: $N=2,3$}

In the case of only two overlapping resonances, integration over their energies results in the following joint widths $x, y$ distribution

$$
\begin{aligned}
& \Phi(x, y ; \kappa)=\frac{\pi}{32} \kappa^{2} \exp \left(\frac{\pi}{32} \kappa^{2}(x+y)^{2}\right) \\
& \quad \times\left[\left(x^{2}-6 x y+y^{2}\right) K_{0}\left(\frac{\pi}{32} \kappa^{2}(x+y)^{2}\right)\right. \\
& \left.\quad+(x+y)^{2} K_{1}\left(\frac{\pi}{32} \kappa^{2}(x+y)^{2}\right)\right] \\
& \quad \times \exp \left(-\frac{\pi}{4} \kappa^{2} x y\right),
\end{aligned}
$$

where $K_{n}$ stands for the Macdonald function. Note that this expression is fully symmetric with respect to the two widths. Final integration over one of the widths

$$
\Psi(x ; \kappa)=\int_{0}^{\infty} \Phi(x, y ; \kappa) \frac{\mathrm{e}^{-\frac{y}{2}}}{\sqrt{2 \pi y}} \mathrm{~d} y
$$

has been performed numerically (see Fig.1, upper panel). The effect of overlapping turns out to be almost two times stronger in the case of three resonances (see Fig.1, lower panel).

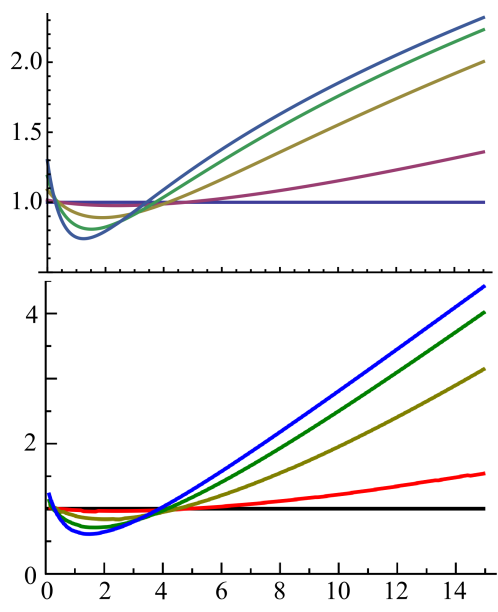

Fig. 1. $\Psi(x ; \kappa)$. Two and three resonances in the upper and lower panel, respectively for (from bottom to top in the right hand side) $\kappa=0,0.1,0.3,0.5,0.7$.

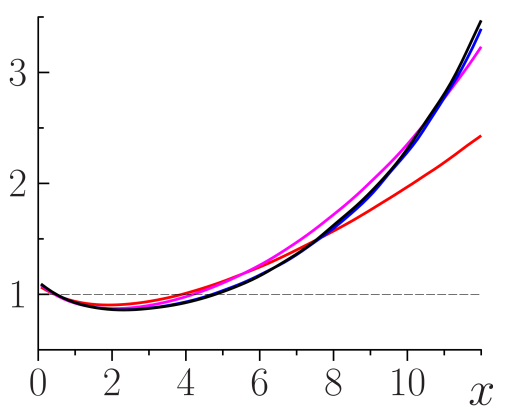

Fig. 2. $\Psi(x ; \kappa=0.2)$ for (from bottom to top for $\mathrm{x}=12) N=3,5,80,100$.

However, if the number $N$ of resonances is large enough the influence of distant ones diminishes quite rapidly so that the principal effect is due to some restricted number of near neighbors (see Fig.2, numerical calculation).

\section{Summary}

The principal point of our "local" approach rely on the idea to focus attention on a finite intervals of the total energy spectrum within which the mean level spacing remains constant. Just the basic eq.(1), that is valid for any finite number of resonances, affords the necessary adequate tool. We have explicitly demonstrated that only a relatively small number of neighboring resonances essentially influence on the widths distribution of (arbitrarily selected and therefore typical) one of our interest. 


\section{Acknowledgments}

I am very grateful to L. Sirko for his interest in this work. I am also obliged to Oleg V. Zhirov for numerous discussions and accomplishing numerical calculations.

\section{References}

[1] P.E. Koehler, F. Bečvář, M. Krtička, J. A. Harvey, K.H. Guber, Phys. Rev. Lett. 105, 072502 (2010); P.E. Koehler, Phys. Rev. C 84, 034312 (2011); P.E. Koehler, F. Bečvář, M. Krtička, K.H. Guber, J.L. Ullmann, Fortschritte der Physik 61, 80 (2012).
[2] V.V. Sokolov, V.G. Zelevinsky, Nucl. Phys. A 504, 562 (1989); V.V. Sokolov, V.G. Zelevinsky, Phys. Lett. B 202, 10 (1988).

[3] M.L. Mehta, Nucl.Phys. 18, 395 (1960).

[4] G. Shchedrin, V. Zelevinsky, Phys. Rev. C 86, 044602 (2012).

[5] Y.V. Fyodorov, D.V. Savin, EPL 110, 40006 (2015).

[6] A. Volya, H.A. Weidenmüller, V. Zelevinsky, Phys. Rev. Lett. 115, 052501 (2015).

[7] E. Bogomolny, Phys. Rev. Lett. 118, 022501 (2017). 